\title{
Nitro-PAK aus Verbrennungsmotoren
}

\section{- Analytik und Emissionsverhalten*}

\author{
Heidelore Fiedler ${ }^{1}$, W. Mücke ${ }^{2}$ \\ 1 Lehrstuhl für Ökologische Chemie und Geochemie, Universität Bayreuth, Postfach 1012 51, W-8580 Bayreuth \\ 2 Bayerisches Staatsministerium für Landesentwicklung und Umweltfragen, Rosenkavalierplatz 2, W-8000 München 81 und Ludwig-Maximilians- \\ Universität München
}

Zusammenfassung. Eine VDI-Vorschrift zur Messung polycyclischer aromatischer Kohlenwasserstoffe (PAK) und damit auch von Nitro-PAK existiert z.Zt. noch nicht. Für die Probenahme wird in der Regel die Vollstrommethode oder die Verdünnungsmethode verwendet. Analytische Aufarbeitung, Extraktion und Fraktionierung richten sich nach der Komplexität der Stoffgemische. Für die Auftrennung kommen Gaschromatographie und Hochdruckflüssigkeits-Chromatographie in Frage, die Identifizierung erfolgt mittels Massenspektroskopie und selektiver Detektoren.

Zum Emissionsverhalten ist festzustellen, daß mangels valider Daten vorerst auf Ergebnisse von PAK zurückgegriffen werden muß, um die Nitro-PAK abzuschätzen. Vermutlich werden die NitroPAK in der Nachbrennphase gebildet. Eine Verminderung scheint durch eine Variation der Betriebsparameter und die Verwendung des 3-Wege-Katalysators möglich.

\section{Einleitung}

Die Grundbedingung exakter Analytik von Umweltchemikalien in komplexen Probenmatrices ist eine Probenahme, die das gesammelte Probengut sowohl in qualitativer als auch quantitativer Hinsicht so wenig wie möglich beeinträchtigt. Um Exaktheit zu garantieren, ist die Probenahme gerade bei der Erfassung von Motoremissionen aufwendig. Erschwerend kommt hinzu, daß es bei nicht-limitierten $\mathrm{Ab}$ gaskomponenten keine standardisierten Verfahren gibt. Probenahmetechniken und -ausrüstungen sind Modifikationen der Verfahren, die für Müllverbrennungsanlagen entwickelt worden sind. Die Analytik selbst vollzieht sich in den Schritten Extraktion, Fraktionierung, Detektion und Identifizierung.

\section{Probenahme}

Zur Untersuchung von Nitro-PAK im Abgas von Verbrennungsmotoren sind im wesentlichen zwei Verfahren üblich:

\footnotetext{
"Nitro-PAK - Bildung, Umweltverhalten und Wirkungen, siehe UWSF 2 (3) $170-176(1990)$
}

- Vollstrommethode: Mit diesem Verfahren (GRIMMER et al., 1972; KRAFT und LIES 1981) erfaßt man das gesamte erzeugte Abgas, indem das Abgas in einer Sammelvorrichtung kondensiert und filtriert wird.

- Verdünnungsmethode: Dieses Verfahren wird von der U.S. Environmental Protection Agency (EPA) für die Bestimmung von Partikelemissionen aus Dieselmotoren vorgeschrieben. Hierbei werden die an Partikel adsorbierten Nitro-PAK aus einem bereits vorher mit filtrierter Frischluft verdünnten Teilstrom filtriert. Ergebnisse, die mit dieser Methode erhalten wurden, finden sich bei Hartung et al. (1982) und Lies (1986). Der Entwurf einer VDI-Vorschrift zur Messung polycyclischer aromatischer Kohlenwasserstoffe sieht ebenfalls die Anwendung der Verdünnungsmethode vor (VDI, 1989).

Eine Probenahmevorrichtung, die es erlaubt, Schadstoffkonzentrationen direkt im Verbrennungsraum zu erfassen, ist von HaYano et al. (1985) beschrieben worden. Das Prinzip beruint darauf, daß ein Ventil direkt in den Verbrennungsraum eingeführt wird und so Aliquots bei den hohen Verbrennungstemperaturen (unter Druck) entnommen werden. Diese Vorrichtung wäre geeignet, endgültig zu klären, ob Nitro-PAK bereits im Verbrennungsraum entstehen oder erst im Auslaßsystem. Die Werte der PAKMessungen direkt im Verbrennungsraum lagen um 2-3 Größenordnungen über denen im Abgas (das den Auspuff verläßt); die mutagene Aktivität in den Abgaspartikeln war um eine Größenordnung niedriger als die im Verbrennungsraum.

\section{Artefaktbildung}

Auf Filtermaterial aufgebrachte, reine, gelöste PAK reagieren mit $\mathrm{NO}_{2}$ im Zuge elektrophiler aromatischer Reaktionen zu entsprechenden Nitroderivaten (PITTS et al., 1978; SChUetzle 1983). Falls dieses auch für an Partikel adsorbierte PAK zuträfe, würden damit die Konzentrationen an Nitro-PAK im Abgas von Motoren vor allem bei längeren Sammelzeiten überschätzt. Erste Belege für derlei Artefakte, die schon vorher vermutet worden waren (GIBSON et al., 1981), sind mittels stichhaltiger ausgedehnter Erhebun- 
gen bestätigt worden (HARTUNG et al., 1984; HARTUNG et al., 1986).

Für Probenahmezeiten von weniger als 45 Minuten liegt die Artefaktbildung für 1-Nitropyren bei Sammelbedingungen, die den US-Vorschriften (Federal Test Procedure) entsprechen, bei weniger als $20 \%$ des an Partikeln adsorbierten 1-Nitropyren. Die Primärquelle für 1-Nitropyren im Dieselabgas ist demnach der Bildungsproze $\beta$ im Motor und im Auslaßsystem. Für andere Nitro-PAK kann ähnliches vermutet werden.

Die Artefaktbildung ist abhängig von der Reaktivität der Ausgangs-PAK in elektrophilen aromatischen Reaktionen. Tabelle 1 zeigt eine Zusammenstellung der normalerweise in Dieselabgas gefundenen PAK, abgestuft nach ihrer Reaktivität in elektrophilen aromatischen Reaktionen.

Tabelle 1: Klassifizierung der PAK-Reaktivität in elektrophilen aromatischen Reaktionen; Ordnung der PAK nach abnehmender Reaktivität (NiELSEN et al., 1986)

Gruppe 1: Benzo[a]tetracen, Pentacen, Tetracen

Gruppe 2: Anthracen, Anthantren, Benzolalpyren, Perylen

Gruppe 3: Benz[a]anthracen, Benzo[ghi]perylen, Cyclopenteno[c,d]pyren, Pyren

Gruppe 4: Benzo[c]phenanthren, Benzo[e]pyren, Chrysen, Coronen, Dibenzanthracene

Gruppe 5: Benzofluoranthene, Fluoranthen, Triphenylen, Indeno[1,2,3,-c,d]pyren, Naphthalin, Phenanthren

Die Reaktivität der im Motor gebildeten PAK hängt von der Temperatur im Probenahmesystem ab. Um die während des Verbrennungsprozesses gebildeten Nitro-PAK exakt quantifizieren zu können, muß das heiße Abgas nach Austritt aus der Auspuffanlage sofort gekühlt werden. So werden elektrophile Reaktionen der PAK mit reaktiven Gasen, die zur Artefaktbildung führen, auf ein Minimum beschränkt.

\section{Analytische Aufarbeitung von Umweltproben}

\subsection{Extraktion und Fraktionierung}

Die Erfassung der Nitro-PAK beginnt mit der Extraktion aus der Probenmatrix und nachfolgend der Einengung des Extraktionsmittels am Rotationsverdampfer, wobei zur Quantifizierung vor der Extraktion ein interner Standard zugesetzt wird.

Zur Extraktion werden binäre Lösungsmittelsysteme verwendet (KOPCZYNSKI 1987): entweder eine aromatische Komponente (Toluol oder Xylol) in Verbindung mit einem Alkohol (z.B. das System Toluol $/$ Propanol $=51 / 49)$ oder Aromaten in Verbindung mit Dichlormethan (Toluol/Dichlormethan $=3 / 1$ ).

Die chemische Zusammensetzung der Partikel, z.B. in Dieselabgasen, ist äußerst komplex. Die organischen Extrakte bestehen aus aliphatischen Kohlenwasserstoffen, PAK, Nitro-PAK, N- und S-Heterocyclen und einer. Vielzahl anderer organischer Verbindungen. Um einzelne Klassen von Verbindungen oder gar individuelle Spezies analysieren zu können, muß der Lösungsmittelextrakt einer Fraktionierung unterworfen werden. Dies kann säulenchromatographisch in Florisil- oder Kieselgelsäulen durch Elutionsmittel unterschiedlicher Polarität erfolgen (KOPCZYNSKI 1987; JIN und RAPPAPORT 1983).

Eine andere Methode der Fraktionierung besteht in der präparativen Auftrennung mittels Hochdruckflüssigkeits-Chromatographie (HPLC). Durch geeignete Wahl des Säulenmaterials und Verwendung von Druck- und/oder Elutionsmittelgradientenprogrammen kann bereits eine sehr umfassende Auftrennung des Extraktes erfolgen (LIBERTI et al., 1984).

\subsection{Detektion und Identifizierung}

Die analytische Endphase besteht in der chromatographischen Auftrennung und der Detektion der Verbindungen.

Als chromatographische Verfahren kommen Gaschromatographie, Hochdruckflüssigkeits-Chromatographie und Hochleistungs-Dünnschichtchromatographie in Frage. Zur Detektion kommen stickstoffselektive Detektoren (NSD), Elektroneneinfangdetektoren (ECD), Flammenionisationsdetektoren (FID), Wärmeleitfähigkeitsdetektoren (WLD), Massenspektrometrie (MS) und solche Detektoren in Frage, die mittels Fluoreszenz, Chemilumineszenz oder elektrochemischer Verfahren arbeiten. Die einzelnen Methoden unterscheiden sich hinsichtlich ihrer Selektivität und Sensitivität. Je nach Fragestellung ist das geeignete Verfahren auszuwählen und zu testen. Eine weitere Verfeinerung der chromatographischen Auftrennung und nachfolgender Detektion ist durch Hintereinanderschaltung gleicher oder verschiedener chromatographischer Verfahren und geeignete Kombination mehrerer Detektoren möglich. Solche Verfahren werden als multi-dimensional bezeichnet. Die Abb. zeigt beispielhaft das Gaschromatogramm eines Dieselextraktes mit $\mathrm{N}$-selektivem Detektor:

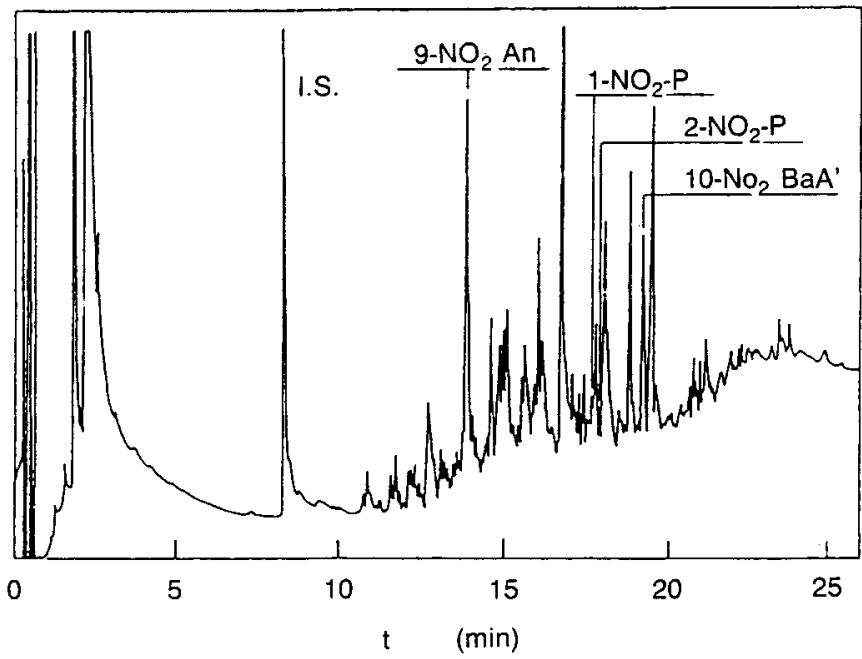

Abb.: Gaschromatogramm eines Dieselabgasextraktes, detektiert mit stickstoffselektivem Detektor (NPD) nach vorheriger StoffFraktionierung mittels HPLC (LiBERTI et al., 1984)

Durch Verfeinerung der Methoden werden immer geringere Mengen an Nitro-PAK zuverlässig detektiert und quanti- 
fiziert. So erreichte man mit einem multi-dimensionalen gaschromatographischen System und stickstoffselektiver Detektion für 1-Nitropyren eine Nachweisgrenze von $36 \mathrm{pg}$ (Pikogramm $=10^{-12} \mathrm{~g}$ ), die zudem hochreproduzierbar war (KoPCZINSKI 1987). Mit Hilfe multi-dimensionaler HPLC und anschließender Fluoreszenzdetektion für 1-Nitropyren nach dessen katalytischer Reduktion zu dem entsprechenden aromatischen Amin konnte die Nachweisgrenze sogar auf 10 pg herabgesetzt werden (TEJADA et al., 1986).

Zur Identifizierung der Verbindungen ist eine Koppelung Gaschromatographie-Massenspektrometrie (GC-MS) oder Massenspektrometrie-Massenspektrometrie (MS-MS) geeignet (HENDERSON et al., 1982; HENDERSON et al., 1983; SCHuETZle et al., 1982). Der Nachteil der MS/MS Kopplung ist jedoch, daß keine Isomeren einer Verbindung unterschieden werden können. Anweisungen zur Synthese von einigen Nitro-PAK, die in der Analytik zur Quantifizierung der Ergebnisse verwender werden können, finden sich bei BODINE et al. (1981). Eine Übersicht über die Eigenschaften einiger in der Routineanalytik gebräuchlicher Methoden zeigt Tabelle 2 .

Tabelle 2: Analytische Methoden zur Bestimmung von Nitro-PAK

\begin{tabular}{|c|c|c|c|c|}
\hline \multicolumn{2}{|c|}{ Methode Säule } & \multirow{2}{*}{$\begin{array}{l}\text { Detektor } \\
\text { N-selektiv }\end{array}$} & \multirow{2}{*}{$\begin{array}{c}\begin{array}{c}\text { Detektions- } \\
\text { grenze }\end{array} \\
0,1-1\end{array}$} & \multirow{2}{*}{$\begin{array}{l}\text { Bemerkungen } \\
\begin{array}{l}\text { Wenige Interfe- } \\
\text { renzen }\end{array}\end{array}$} \\
\hline $\mathrm{GC}$ & Gepackt & & & \\
\hline & Kapillar & N-selektiv & $0,1-1$ & $\begin{array}{l}\text { N/P-Selektivität } \\
10^{4}\end{array}$ \\
\hline & Gepackt & ECD & $0,01-0,1$ & $\begin{array}{l}\text { Interferenz mit } \\
\text { Halogenen }\end{array}$ \\
\hline & Kapillar & ECD & $0,01-0,1$ & \\
\hline & Gepackt & TEA & 0,1 & $\begin{array}{l}\text { Gut für Nitros- } \\
\text { amine }\end{array}$ \\
\hline & Gepackt & $\begin{array}{l}\text { Elektr. } \\
\text { Leitfähigkeit }\end{array}$ & 0,1 & $\begin{array}{l}\text { Nur für } \mathrm{NO}_{2}^{-} \\
\text {Verbindungen }\end{array}$ \\
\hline \multirow[t]{3}{*}{ HPLC } & Gepackt & Fluoreszenz & $0,01-1$ & $\begin{array}{l}\text { Reversed/normal } \\
\text { Phase }\end{array}$ \\
\hline & Gepackt & Elektrochemisch & $0,1-1$ & $\begin{array}{l}\text { Nur reversed } \\
\text { Phase }\end{array}$ \\
\hline & Gepackt & TEA & 0,1 & \\
\hline
\end{tabular}

TEA = Thermal Energy Analyzer; ECD = Electron Capture Detector (Elektroneneinfangdetektor)

\section{Emissionen aus Verbrennungsmotoren}

Die im Abgasstrom von Verbrennungsmotoren enthaltenen PAK treten hauptsächlich partikelgebunden auf. Mehr als 20 Nitro-PAK sind in Dieselabgasen nachgewiesen worden. 1-Nitropyren hat mit Gehalten zwischen 107 und 1590 ppm (relativ zum Gewicht des Extrakts) den größten Anteil, gefolgt von Nitrophenanthren/Anthracen-Isomeren (LEWTAS und WILliams 1986). Dinitropyrenisomere wurden in einer Probe im Sub-ppm-Bereich $(0,4-0,6 \mathrm{ppm})$ identifiziert. Trotz des hohen Anteils von 1-Nitropyren im Dieselextrakt macht diese Substanz jedoch nur $3-13 \%$ der Mutagenität aus. Die mit 0,4-0,6 ppm (Summe = 1,6 ppm) nur geringen Mengen an Dinitropyrenisomeren
$(1,3,-1,6,-1,8)$ sind dagegen für ca. $26 \%$ der Mutagenität dieser Probe verantwortlich ( $\rightarrow$ Tabelle 3 ).

Tabelle 3: Beitrag von Nitro-PAK zur Mutagenität von partikelgebundenen Extrakten in Salmonella typhimurium TA98 (-S9) (LEWTAS und WILLIAMSON 1986)

\begin{tabular}{|c|c|c|c|c|c|c|c|}
\hline \multirow{2}{*}{$\begin{array}{l}\text { Abgas- } \\
\text { extrakt } \\
\text { Probe }\end{array}$} & \multirow{2}{*}{$\begin{array}{c}\text { Mutagene } \\
\text { Aktivität } \\
\text { (Rev/ } \mu \mathrm{g}) \\
\text { TA98-S9 }\end{array}$} & \multicolumn{2}{|c|}{$\begin{array}{l}\text { 1-Nitro- } \\
\text { pyren }\end{array}$} & \multicolumn{2}{|c|}{$\begin{array}{l}\text { Nitrofluor- } \\
\text { anthen }\end{array}$} & \multicolumn{2}{|c|}{$\begin{array}{c}\text { Dinitropyren } \\
\text { Isomere }\end{array}$} \\
\hline & & $(\mathrm{ppm})$ & $(\%)$ & (ppm) & $(\%)$ & (ppm) & $(\%)$ \\
\hline \multirow[t]{3}{*}{ Diesel } & 13,0 & 1590 & 11,0 & 7,0 & 1,4 & - & \\
\hline & 3,9 & 589 & 13,0 & 1,2 & 0,8 & 1,6 & 26,0 \\
\hline & 3,5 & 107 & 2,7 & 0,9 & 0,8 & - & - \\
\hline Benzin & 1,6 & 2,5 & 0,1 & - & - & - & - \\
\hline
\end{tabular}

Da einerseits erst wenige Erfahrungen mit Nitro-PAK hinsichtlich ihrer direkten Entstehung im Motor in Abhängigkeit verschiedener Parameter vorliegen, andererseits davon ausgegangen werden kann, daß Nitro-PAK durch Nitrierungsreaktionen von $P A K$ entstehen, muß auf einschlägige Ergebnisse von Untersuchungen der PAK-Bildung im Motor zurückgegriffen werden.

Obwohl eine Vielzahl von PAK in Automobilabgasen nachgewiesen wurden, werden bei Routinemessungen nur wenige bestimmt (der Verzicht bringt einen erheblichen Zeitgewinn bei der Analyse und vereinfacht das Clean-up). In Autoabgasen wurden bei Anwendung der Verdünnungsmethode bis zu $200 \mu \mathrm{g} /$ Meile an Fluoranthen und Pyren, ca. $20 \mu \mathrm{g} /$ Meile an Indenopyren, Benzo[ghi]perylen, Coronen und $1 \mu \mathrm{g} /$ Meile Perylen gefunden (LIES et al., 1986).

Als Gesamtaussage ergibt sich, daß alle Kraftfahrzeuge, unabhängig von der Art des Kraftstoffs, dasselbe typische $P A K$-Muster emittieren. Für eine Gefahrenabschätzung muß berücksichtigt werden, daß $80 \%$ der emittierten PAK aus Fluoranthen und Pyren bestchen, die hinsichtlich der Gesundheitsgefährdung vernachlässigt werden können (LIES et al., 1986).

In einer umfangreichen Studie (LIES et al., 1988) wurden Abgastests nach drei verschiedenen Testverfahren auf Rollenprüfständen durchgeführt: FTP (Federal Test Procedure nach US-Test 75), SET (US-Sulfat-Emissionstest) und HDC (US-Highway-Driving Test = Highway Fuel Economy Driving Cycle).

Die Massenanteile der Partikelemissionen verhielten sich für die drei Fahrzeugtypen Ottomotor ohne Katalysator: Ottomotor mit Katalysator: Dieselmotor wie $7: 3: 100$ (LIES et al., 1988). Die insgesamt niedrigen Konzentrationen der Katalysatorfahrzeuge sind im wesentlichen auf die Abwesenheit von Bleiverbindungen im Kraftstoff zurückzuführen.

Bei den PAK-Emissionen zeigt es sich, daß Ottomotorfahrzeuge mit Katalysator bei allen 11 untersuchten Substanzen um mindestens eine Größenordnung niedrigere PAKKonzentrationen emittieren als Fahrzeuge mit Ottomotor ohne Katalysator und Dieselmotoren. Eine Abhängigkeit vom Testcyclus oder vom Motortyp (Zylinderzahl) ist nicht 
erkennbar. Die Absenkung mit dem Katalysatorkonzept liegt zwischen 89 und $96 \%$ (LIES 1988). Im Mittel verhalten sich die emittierten PAK-Mengen bei den drei Fahrzeuggruppen (Ottomotor ohne Katalysator, Ottomotor mit Katalysator, Dieselmotor) wie 62:4:100 $\rightarrow$ Tabelle 4).

Tabelle 4: Emissionsgesamtmittelwerte für 11 polycyclische aromatische Kohlenwasserstoffe, gebildet über die drei Testverfahren und alle Fahrzeuge je Fahrzeuggruppe sowie die mittlere Absenkung durch das Katalysatorkonzept (LIES 1988)

\begin{tabular}{lccrc}
\hline Komponente & $\begin{array}{c}\text { Ottomotor } \\
\text { ohne Kat. } \\
(\mu \mathrm{g} / \mathrm{mi})\end{array}$ & $\begin{array}{c}\text { Ottomotor } \\
\text { mit Kat. } \\
(\mu \mathrm{g} / \mathrm{mi})\end{array}$ & $\begin{array}{r}\text { Dieselmotor } \\
(\mu \mathrm{g} / \mathrm{mi})\end{array}$ & $\begin{array}{c}\text { Absenkung } \\
\text { durch Kat. } \\
(\%)\end{array}$ \\
\hline Fluoranthen & 69,7 & 4,4 & 112,0 & 94 \\
Pyren & 69,2 & 4,7 & 107,7 & 93 \\
Benz[a]anthracen & 8,3 & 0,3 & 5, & 96 \\
Chrysen & 13,5 & 0,6 & 20,8 & 96 \\
Benzo[b/k] & & & & \\
$\quad$ fluoranthen & 10,4 & 0,8 & 17,1 & 92 \\
Benzo[e]pyren & 8,3 & 0,4 & 24,7 & 95 \\
Benzo[a]pyren & 6,3 & 0,4 & 7,6 & 95 \\
Perylen & 1,5 & 0,1 & 0,9 & 93 \\
Indeno[1,2,3-cd] & & & & 89 \\
$\quad$ pyren & 3,8 & 0,4 & 6,3 & 89 \\
Benzo[ghi]perylen & 9,7 & 0,6 & 16,7 & 94 \\
\hline Summe & 196,0 & 12,5 & 315,0 & 94 \\
\hline
\end{tabular}

Benzo[b/k]fluoranthen = Benzo[b]fluoranthen + Benzo[k]fluoranthen

Eine detaillierte Studie über die Bildung von PAK in Abhängigkeit verschiedener Motorbetriebsparameter (LEPPERHOF 1981) untersucht das Luft-Kraftstoffverhältnis, die Last, den Zündzeitpunkt und Kühlwassertemperatur sowie die Beeinflussung des Polycyclenausstoßes durch das verwendete Schmieröl und durch Brennraumablagerungen. Die PAK entstehen nicht nur direkt im Verbrennungsraum, durch Oxidations-, Crack- und Pyrolysereaktionen können auch während der Expansions- und Ausschiebephase PAK gebildet werden. Ein Nebeneffekt der PAK-Bildung im Motor ist die PAK-Kumulierung im Motorenöl mit zunehmender Gebrauchszeit (SHORE und OCKERT 1958; BEGEMAN und ColucCl 1970; Gross 1972; Heitland et al., 1976; LEPPERHOF 1981; BERGNER 1982; LEPPERHOF 1982; ROSEN. KRANZ und MermelsteIN 1983; SCHUetZle und FraZier 1986; WENTWORTH, undatiert).

Zur Relevanz der Ergebnisse der PAK-Emission für die Emission von Nitro-PAK ist kritisch festzustellen: Einerseits liegen noch keine Erkenntnisse über die direkte Entstehung von Nitro-PAK im Motor in Abhängigkeit verschiedener Parameter vor; andererseits kann davon ausgegangen weerden, daß Nitro-PAK durch Nitrierungsreaktionen von PAK entstehen, so daß auf einschlägige Ergebnisse von Untersuchungen der PAK-Bildung im Motor zurückgegriffen werden kann.

Vermutlich werden die Nitro-PAK nicht im Motor selbst gebildet, sondern erst in der Nachverbrennungsphase bei gleichzeitiger Anwesenheit von PAK, Stickstoffoxiden und Spuren von Säuren, die als Katalysator wirken (ROSENKRANZ und MERMELSTEIN 1983). Geht man davon aus, daß Nitro-PAK nur durch Nitrierung von PAK gebildet werden, muß also erst das PAK über radikalische Polymerisationsprozesse kurzkettiger Kohlenwasserstoffspezies gebildet werden. Das bedeutet, daß ein Großteil der Reaktionszeit für die Synthese des PAK benötigt wird (RISBY und LESTZ 1983). Jedoch könnte es auch sein, daß radikalische $\mathrm{Zwi-}$ schenprodukte bereits vor dem Ringschluß nitriert werden (RISBY und LESTZ 1983).

Eine Verminderung der Nitro-PAK-Bildung scheint möglich durch:

- eine Variation der Motorbetriebsparameter, die zu einem geringeren PAK-Ausstoß führen. Dazu kommen in Betracht: Betrieb mit vorwiegend mageren Gemischen, geringe Spaltvolumina im Brennraum und Erhöhung der Zylinderwandtemperaturen.

- den 3-Wege-Katalysator.

\section{Literatur}

Begeman, C. R.; J. M. ColuCCI: Polynuclear aromatic hydrocarbon emission from automotive engines. SAE Trans. 79 (1970)

Bergner, P.: Zur Polycyclenbildung im Ottomotor bei der Verbrennung von Kohlenwasserstoffen unter variierten motorischen Betriebsbedingungen. Dissertation, Technische Universität München (1982)

BODINE, R. S.; P. H. RueHLE; R. W. ROTH; G. BosCH; L. BoSCH; G. OppermanN; J. H. Saugier: Synthesis of selected nitro polycyclic aromatic hydrocarbons. Midwest Research Institute, 425 Volker Boulevard, Kansas City, Missouri 64110, USA (1981)

Gibson, T. L.; A. I. RICCl; R. L. WILliams: Measurement of PAH, their derivarives, and their reactivity in diesel automobile exhaust; 707 ff. In: Bjorrseth, A.; Dennis, A. J.; Eds.: Polynuclear Aromatic Hydrocarbons. Battelle Press, Columbus, Ohio. (1981)

Grimmer, G.; A. Hit.derrandt; H. Bornnke: Probenahme und Analycik polycyclischer aromatischer Kohlenwasserstoffe in Kraftfahrzeugabgasen. Erdöl und Kohle-Erdgas, Petrochemie 25, 442 (1972)

Gross, G. P.: The effect of fuel and vehicle variables on polynuclear aromatic hydrocarbons and phenol emissions. SAE-Paper 720210 (1972)

Hartung, A.; J. KRafT; K.-H. Lies; J. Schulze J.: Messung polycyclischer aromatischer Kohlenwasserstoffe im Abgas von Dieselmotoren. MTZ 43, S. 263 (1982)

Hartung, A.; J. KrafT; J. Schulze; H. Kieß; K.-H. Lies: The identification of nitrated PAH in diesel particulate extracts. Chromatographia 19, 269 (1984)

HARTUNG, A. J.; J. SCHULZE; H. KiEß;; K.-H. LiES: Nitroderivate der PAK als Artefakte bei der Probenahme aus dem Dieselabgas. Staub-Reinhaltung der Luft 46, 132 (1986)

Hayano, S.; L. JaNG-Ho; K. FuRuYa; T. KikUCHI; T. SOMEya; Ch. OIKawa; Y. Lida; H. Matsushita; T. KinouChi; Y. ManaBE; Y. OHNISHI: Formation of hazardous substances and mutagenicity of $\mathrm{PAH}$ produced during the combustion process in a diesel engine. Atmospheric Environment 19, 1009 (1985)

Heitland, H.; P. BernhardT; P. Heidemeyer; W. Behrens: Ermittlung polycyclischer aromatischer Kohlenwasserstoffe im Automobilabgas in Abhängigkeit vom Motorkonzept und Fahrzustand. VW-Forschungsbericht Nr. F2 - 76/19 (1976)

Henderson, T. R.; R. E. Royer; Ch. R. Clark; T. M. Harvey; D. F. HUNT: MS/MS analyses of diesel emissions and fuels treated with $\mathrm{NO}_{2}$. J. Applied Toxicology 2, 231 (1982)

Henderson, T. R.; J. D. Sun; R. E. Royer; Ch. R. Clark; A. P. LeE; T. M. HarveY; D. F. Hunt; J. E. Fulford; A. M. Lovetre; W. R. Davidson: Triple-quadrupole mass spectrometry studies of nitroaromatic emissions from different diesel engines. Environ. Sci. Technol. 17, 443 (1983)

JIN, Z.; S. M. RAPPAPORT: Microbore liquid chromatography with electrochemical detection for determination of nitro-substituted 
polynuclear aromatic hydrocarbons in diesel soot. Anal. Chem. 55,1778 (1983)

KOPCZYNSKI, S. L.: Application of multidimensional gas chromatography to analysis for nitrated polycyclic aromatic hydrocarbons in airborne particulate matter. Intern. J. Environ. Anal. Chem. 30, (1987)

KRAFT J.; K.-H. LIES (1981): Polycyclic aromatic hydrocarbons in the exhaust of gasoline and diesel vehicle. SAE-Paper No. 810082 (1981)

LEPPERHOF, G: PAH-Emissionen von Ottomotoren (1. Mitt.). Wissenschaft und Umwelt 2, 72 (1981)

Op. cit.: 1, 1 (1982)

LETWAS, J; K. WILLIAMSON: A retrospective view of the value of short-term genetic bioassays in predicting the chronic effects of diesel soot. In: Carcinogenic and Mutagenic Effects of Diesel Engine Exhaust, 119 ff. N. Ishinishi; A. KolZumi; R. O. MC Clellan; W. STOOBER (Hrsg.) Elsevier Science Publishers, Amsterdam-New York-Oxford (1986)

Liberti, A.; P. Cicioli; A. Cecinato; E. Brancaleoni; C. D. Palo: Determination of nitrated-polyaromatic hydrocarbons (nitroPAHs) in environmental samples by high resolution chromatographic techniques. J. High Res. Chromatogr. Commun. 7, 389 (1984)

Lies, K. H.; A. Hartung; A. Postulka; H. Gring; J. Schulze: Composition of diesel exhaust with particular reference to particle bound organics including formation of artifacts. In: Carcinogenic and Mutagenic Effects of Diesel Engine Exhaust, $65 \mathrm{ff}$. N. IsHINIS HI; A. Kolzumi; R. O. MC ClellaA; W. STÖBER (Hrsg.) Elsevier Science Publishers, Amsterdam - New York-Oxford (1986)

Lies, K. H.; J. SChUlze; H. WinNeKe; M. KuhleR; J. Kraft; A. HartunG; A. Postulka; H. Gring; D. Schröter: Nicht limitierte Automobil-Abgaskomponenten. Volkswagen AG, Wolfsburg (1988)

Pitts, J. N. Jr.; K.A. van Cauwenherghe,; D. Grosjean; J. P. SChmid; J. P. Fitz; W. L. Besier, Jr.; G. B. KNudson; P. M. HYNDS: Atmospheric reactions of polycyclic aromatic hy- drocarbons: Facile formation of mutagenic nitro derivatives. Science 202,515 (1978)

RISBY, T. H.; S. S. LESTZ: Is the direct mutagenic activity of diesel particulate matter a sampling artifact? Environ. Sci. Technol. 17, 621 (1983)

Rosenkranz, H. S.; R. Mermelstein: Mutagenicity and genotoxicity of nitroarenes - all nitrocontaining chemicals were not created equal. Mutat. Res. 114, 217 (1983)

SCHUETZle, D.: Sampling of vehicle emissions for chemical analysis and biological testing. Environ. Health Perspect 47, 65 (1983)

Schuetzle, D.; T. L. Riley; T. J. Prater: Analysis of nitrated polycyclic aromatic hydrocarbons in diesel particulates. Anal. Chem. 54,265 (1982)

SCHUETZLE, D.; J. A. FRAZIER: Factors influencing the emission of vapor and particulate phase components from diesel engines. In: Carcinogenic and Mutagenic Effects of Diesel Engine Exhaust, $41 \mathrm{ff}$. N. Ishinishi; A. KoIZlimi; R. O. MC Clellan; W. STÖber, W. (Hrg.), Elsevier Science Publishers, Amsterdam - New YorkOxford (1986)

SHORE, L. B.; K. F. OCKERT: Combustion chamber deposits - a radiotracer study. SAE Trans. 66, 285 (1958)

TejadA, S. B.; R. B. Zweidinger; J. E. SigSby, Jt.: Fluorescence detection and identification of nitro derivatives of polynuclear aromatic hydrocarbons by on-column catalytic reduction to aromatic amines. Anal. Chem. 58, 1827 (1986)

VDI 3872 Bl. 1: Messen von Emissionen; Messen von polycyclischen aromatischen Kohlenwasserstoffen (PAH); Messen von PAH in Abgasen von Pkw-, Otto- und Dieselmotoren; Gaschromatographische Bestimmung (1989)

VDI 3973BI. 1, Entwurf: Messen von Emissionen; Messen von polycyclischen aromatischen Kohlenwasserstoffen (PAH) an stationären industriellen Anlagen - Verdünnungsmethode; (RWTÜVVerfahren) - Gaschromatographische Bestimmung (1989)

WENTWORTH, J. T.: Effect of combustion chamber surface temperature on exhaust hydrocarbon concentration. SAE-Paper 710587 (undatiert)

\section{Neues aus der EDV}

\section{NETT \\ Network for Environmental Technology Transfer \\ - Das Europäische Informations- instrument für Umweltfragen}

NETT ist eine internationale Vereinigung (ohne Gewinnabsicht) mit Sitz in Brüssel, die mit Unterstützung der EG-Kommission gegründet wurde. Drei Dienstleistungen werden angeboten:

1. Fachinformationen aus On-Line-Datenbanken. Datenbanken von NETT sind über DATANETT zugänglich. DATANETT bietet 4 Bereiche an:
(1) Die Basis-Datenbanken von NETT (NETTBASE, TECHNETT, NETTINFO).

(2) Die Spezialdatenbanken von NETT

- Wasser, Luft, Boden, Lärm, Management

- Umwelt-Gesetzgebung und Europa 1992.

(3) Externe Datenbanken.

(4) Kommunikationsmöglichkeiten (für NETT-Mitglieder untereinander).

2. Persönliche Betreuung individueller Anfragen.

3. "Meeting“-Service und Tagungs-Organisation (Vorträge, Seminare, Teilnahme an Messen).
Die Zahlung eines Jahresbeitrages (EGLänder: $400 \mathrm{ECU} / J a h r ;$ Länder außerhalb der EG: $750 \mathrm{ECU} / \mathrm{Jahr}$ ) vermittelt den Zugang zu den NETT-Dienstleistungen. Die Mitgliedschaft setzt die Tätigkeit in einem der folgenden Umweltbereiche voraus:

- Waren- und Dienstleistungen (Anbieter und Benutzer)

- Forschung und Beratung

- Behörden und Verwaltungen

- Industrieverbände und Handelskammern.

Michel Annez

Secretary General NETT

Ave. Louise 207

B-1050 Brussels

Tel.: 003226450940

Fax: 6464266

\section{"On Line"-Zugriff zu}

EG-Datenbanken

Die Eg hat seit dem 1. April zwei ihrer Datenbanken für die Öffentlichkeit zugänglich gemacht.
1. Mit dem System RAPID können Journalisten und andere Interessierte erstmals an jedem beliebigen Ort in der Welt die täglichen Pressemitteilungen des Sprecherdienstes der Kommission von ihren Terminals aus abrufen.
2. Die Datenbank ECLAS liefert bibliographische Angaben über die Bestände der Zentralbibliothek der EG-Kommission in Brüssel.

Die Redaktion

Quelle: EG-Nachrichten aus München, 6. April 1990 